\title{
Gluonic excitation of non-exotic hybrid charmonium from lattice QCD
}

\author{
Xiang-Qian Luo* and Yan Liu \\ Department of Physics, Zhongshan (Sun Yat-Sen) University, Guangzhou 510275, China
}

(Dated: June 25, 2018)

\begin{abstract}
The ground and first excited states of the hybrid charmonium $\bar{c} c g$, with non-exotic quantum numbers $J^{P C}=0^{-+}, 1^{--}$and $1^{++}$are investigated using quenched lattice QCD. They are completely ignored in the literature, only because their ground states are degenerate with $\eta_{c}, J / \psi$, and $\chi_{c 1}$, and are difficult to be distinguished from these conventional charmonium mesons in experiment. However, we observe strong gluonic radial excitations in the first excited states; We predict that their masses are $4.352(225) \mathrm{GeV}, 4.379(149) \mathrm{GeV}$ and $7.315(257) \mathrm{GeV}$, completely different from the first excited states of the corresponding conventional charmonium. Their relevance to the recent discovery of the $Y(4260)$ state and future experimental search for other states are also discussed.
\end{abstract}

PACS numbers: $12.38 . \mathrm{Gc}, 12.39 . \mathrm{Mk}$

A hybrid meson $\bar{q} q g$ is a bound state of constituent quark $q$, anti-quark $\bar{q}$ and excited gluon $g$. The existence of hybrids is one of the most important predictions of quantum chromodynamics (QCD). There has been a lot of experimental activity [1, 2, 3, 4] in the search for hybrid mesons, for example: PEP-Ш(Babar), KEKB(Belle), 12 $\mathrm{GeV}$ Jefferson Lab upgraded, upgraded CLEO-c detector, and new BES3 detector.

For a conventional meson in the quark model, which is represented by the fermion bilinear $\bar{\psi} \Gamma \psi$, it can have the $J^{P C}$ quantum numbers as $J=|L-S|,|L-S|+1$, ......, $L+S, P=(-1)^{L+1}$, and $C=(-1)^{L+S}$, with $L$ the relative angular momentum of the quark and antiquark, and $S$ the intrinsic spin of the meson. For the gluon, the quantum numbers of the color electric field $\mathbf{E}$ and color magnetic field $\mathbf{B}$ are $1^{--}$and $1^{+-}$respectively. According to QCD, the operator of a hybrid meson is the gauge-invariant direct product of the fermion bilinear $\bar{\psi} \Gamma \psi$ and the color electric field $E_{i}^{c_{1} c_{2}}=F_{0 i}^{c_{1} c_{2}}$ or color magnetic field $B_{i}^{c_{1} c_{2}}=\epsilon_{i j k} F_{j k}^{c_{1} c_{2}}$. Therefore, the quantum numbers of a hybrid meson could be either exotic, with $J^{P C}=1^{-+}, 0^{+-}, 0^{--}, 2^{+-} \ldots \ldots$, inaccessible to conventional mesons, or non-exotic, with $J^{P C}=0^{++}$, $0^{-+}, 1^{--}, 1^{++}, 1^{+-}, 2^{++}, 2^{--}, 2^{-+}, \ldots \ldots$. , the same as conventional mesons.

Lattice gauge theory is the most reliable technique for computing hadron spectra. It involves discretization of the continuum theory on a space-time grid, and reduces to QCD when the lattice spacing goes to zero. The implementation of the Symanzik program $[5]$ with tadpole improvement [6] greatly reduces the discretization errors on very coarse and small lattices. Simulations on anisotropic lattices improve the signal in spectrum computations [7].

The $1^{-+}, 0^{+-}$, and $2^{+-}$exotic hybrid mesons have been extensively studied on the lattice. Reviews can be found in Refs. [8, 9]. Recently, we computed the $0^{--}$ exotic hybrid charmonium mass 10. However, the non-

*Email address: stslxq@zsu.edu.cn exotic hybrid mesons are usually ignored in the literature, simply because their ground states are almost degenerate with the conventional mesons 11 .

In this letter, we investigate the $J^{P C}=0^{-+}, 1^{--}$and $1^{++}$non-exotic charmed hybrid mesons $\bar{c} c g$, employing quenched lattice QCD with tadpole improved gluon [12] and quark [13] actions on the anisotropic lattice. We observe, for the first time, very strong gluonic radial excitations in the first excited states.

Our simulation parameters are listed in Tab. [ We also did simulations on $8^{3} \times 48$ and $12^{3} \times 48$ at $\beta=2.6$, $12^{3} \times 36$ at $\beta=2.8$, and $16^{3} \times 48$ at $\beta=3.0$, but there and throughout the paper we just list the results from the largest volume, i.e., $16^{3} \times 48$ at $\beta=2.6$ and $\beta=$ 2.8 and $20^{3} \times 60$ at $\beta=3.0$. At each $\beta=6 / g^{2}$, three hundred independent configurations were generated with the improved gluonic action [12]. It is also important to check whether these lattice volumes are large enough. When the spatial extent is greater than $2.2 \mathrm{fm}$, the finite volume effect on the spectrum is less than $0.1 \%$ for the ground state, and $0.4 \%$ for the first excited state.

We input the bare quark mass $m_{q 0}$ and then computed quark propagators using the improved quark action [13], the conventional quarkonium correlation function using the operators $0^{-+}=\bar{\psi}^{c} \gamma_{5} \psi^{c}, 1^{--}=\bar{\psi}^{c} \gamma_{j} \psi^{c}$, and $1^{++}=\bar{\psi}^{c} \gamma_{5} \gamma_{j} \psi^{c}$, and the hybrid meson correlation function using the operators $0^{-+}=\epsilon_{i j k} \bar{\psi}^{c_{1}} \gamma_{i} \psi^{c_{2}} F_{j k}^{c_{1} c_{2}}$, $1^{--}=\epsilon_{i j k} \bar{\psi}^{c_{1}} \gamma_{5} \psi^{c_{2}} F_{j k}^{c_{1} c_{2}}$ and $1^{++}=\epsilon_{i j k} \bar{\psi}^{c_{1}} \gamma_{j} \psi^{c_{2}} F_{0 k}^{c_{1} c_{2}}$ in Ref. 14]. Figures [1 and 2 shows the correlation function $C(t)$ of the conventional $1^{--}$and hybrid mesons.

The effective masses of the ground and first excited states $a_{t} m_{1}$ and $a_{t} m_{2}$ are extracted by two different methods: (i) new correlation function method [15]; (ii) modified multi-exponential fit [16]. The multiexponential fitting method has been widely used in the literature 11, 13, 17] for extracting the charmonium masses, and the results for the ground and first excited states are consistent with experiments; The MILC group [16] proposed an improved multi-exponential fitting method, which chooses the best fit according to some criteria. The recently proposed method (i) has been successfully applied to the investigation of the Roper reso- 


\begin{tabular}{|c|c|c|c|c|c|c|c|c|c|c|}
\hline$\beta$ & $\xi=a_{s} / a_{t}$ & $L^{3} \times T$ & $u_{s}$ & $u_{t}$ & $a_{t} r$ & $n_{q 0}$ & $c_{s}$ & $c_{t}$ & $a_{s}\left(1^{1} P_{1}-1 S\right)[\mathrm{fm}]$ & $L a_{s}[\mathrm{fm}]$ \\
\hline 2.6 & 3 & $16^{3} \times 48$ & 0.81921 & 1 & 0.229 & 0.260 & 1.8189 & 2.4414 & $0.1856(84)$ & 2.970 \\
\hline 2.8 & 3 & $16^{3} \times 48$ & 0.83099 & 1 & 0.150 & 0.220 & 1.7427 & 2.4068 & $0.1537(101)$ & 2.459 \\
\hline 3.0 & 3 & $20^{3} \times 60$ & 0.84098 & 1 & 0.020 & 0.100 & 1.6813 & 2.3782 & $0.1128(110)$ & 2.256 \\
\hline
\end{tabular}

TABLE I: Simulation parameters at largest volume. We employed the method in Ref. 13] to tune these parameters, $\kappa_{t}$ and $\kappa_{s}$ for the quark action. The last two columns are about the spatial lattice spacing and the lattice extent in physical units, determined from the $1 P-1 S$ charmonium mass splitting [10], with the effective masses extracted by the method of Ref. [16].

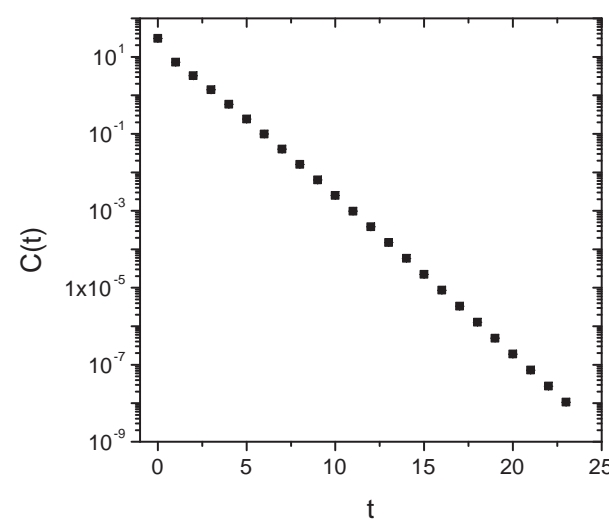

FIG. 1: Correlation function for the conventional $1^{--}$quarkonium at $\beta=2.6$ and $a_{t} m_{q 0}=0.229$.

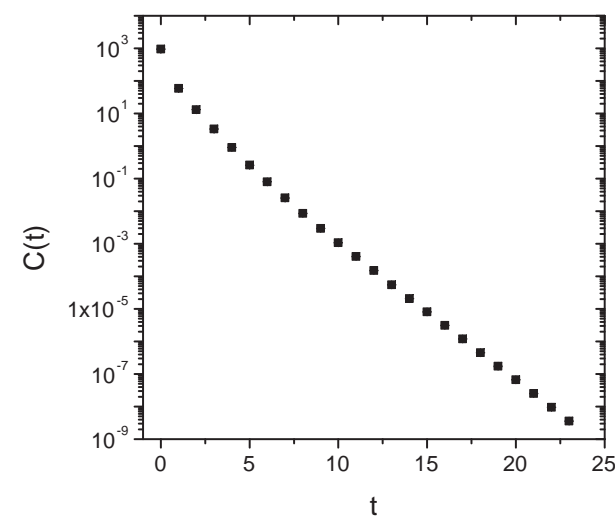

FIG. 2: Same as Fig. 11 but for the $1^{--}$hybrid meson.

nance of the nucleon [15], where $a_{t} m_{1}$ is obtained from $\ln (C(t) / C(t+1))$ in the large time interval $\left[t_{i}, t_{f}\right]$, and $a_{t} m_{1}+a_{t} m_{2}$ from $\ln \left(C^{\prime}(t) / C^{\prime}(t+1)\right)$ in the time interval $\left[t_{i}^{*}, t_{f}^{*}\right]<\left[t_{i}, t_{f}\right]$, with reasonable $\chi^{2} /$ d.o.f. and optimal confidence level. Here $C^{\prime}(t)=C(t+1) C(t-1)-C(t)^{2}$. Two methods provide a cross-check of the results.
Figure [3] shows effective masses for the conventional $1^{--}$quarkonium, where $a_{t} m_{1}$ and $a_{t} m_{1}+a_{t} m_{2}$ are extracted respectively from the plateaux of the lower and upper curves, using the new method [15]. Figure 4 shows those for the $1^{--}$hybrid meson.

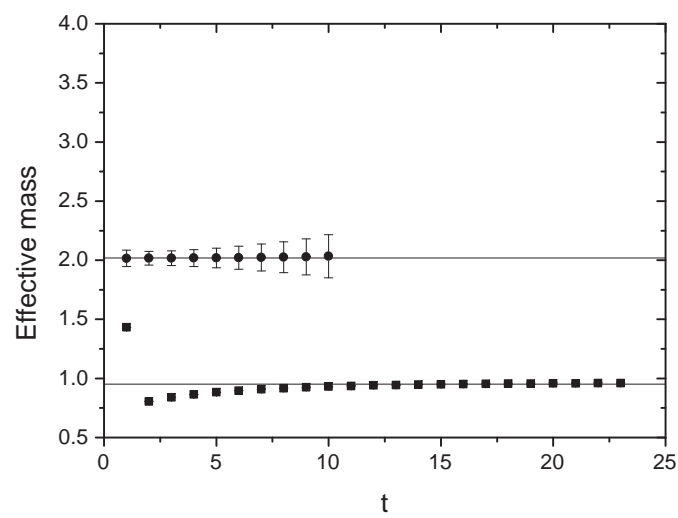

FIG. 3: Effective masses of the conventional $1^{--}$quarkonium as a function of $t$ for $\beta=2.6$ and $a_{t} m_{q 0}=0.229$, using the new correlation function method [15]. $a_{t} m_{1}+a_{t} m_{2}$ and $a_{t} m_{1}$ are extracted respectively from the plateaux of the upper and lower curves, with $\left[t_{i}^{*}, t_{f}^{*}\right]=[1,10]$ and $\left[t_{i}, t_{f}\right]=[11,23]$.

The data at two $m_{q 0}$ values were interpolated to the charm quark regime using $M(1 S)_{\text {exp }}=\left(m\left(\eta_{c}\right)_{\text {exp }}+\right.$ $\left.3 m(J / \psi)_{\text {exp }}\right) / 4=3067.6 \mathrm{MeV}$. The results obtained by the method of Ref. [16] are listed in Tabs. III and III respectively for the ground and first excited states.

The first excited state masses for the conventional $0^{-+}$, $1^{--}$and $1^{++}$charmonium mesons as a function of $a_{s}^{2}$ are plotted in Fig. [5] and those for the hybrid charmonium mesons are plotted in Fig. [6] They indicate the linear dependence of the mass on $a_{s}^{2}$. By linearly extrapolating the data to $a_{s}^{2} \rightarrow 0$, we obtained the spectrum in the continuum limit, which are listed in Tabs. 【and III

As shown in Tab. III in the continuum limit, the masses of the $0^{-+}, 1^{--}$and $1^{++}$charmonium ground states are consistent with their experimental values $2.9804,3.0969$, and 3.5106 for $\eta_{c}(1 S), J / \psi$ and $\chi_{c 1}\left(1^{3} P_{1}\right)$. The results in Tab. II also show that the ground state for the non-exotic hybrid charmonium is degenerate with the 


\begin{tabular}{|c|c|c|c|c|c|c|c|c|}
\hline$\beta$ & $a_{s}^{2}\left(f m^{2}\right)$ & $\eta_{c}$ & $J / \psi$ & $\chi_{c 1}$ & $0^{-+}$ & $1^{--}$ & $1^{++}$ & \\
\hline 2.6 & 0.0345 & $3.031(3)$ & $3.080(3)$ & $3.484(49)$ & $3.012(43)$ & $3.133(44)$ & $3.472(66)$ & \\
\hline 2.8 & 0.0236 & $3.033(1)$ & $3.079(1)$ & $3.446(59)$ & $3.009(51)$ & $3.112(53)$ & $3.463(62)$ & \\
\hline 3.0 & 0.0127 & $3.031(1)$ & $3.080(1)$ & $3.458(64)$ & $3.027(57)$ & $3.099(58)$ & $3.506(65)$ & \\
\hline$\infty$ & 0 & $3.030(2)$ & $3.080(2)$ & $3.430(100)$ & $3.031(87)$ & $3.078(90)$ & $3.516(108)$ & \\
\hline$\infty$ & 0 & $3.053(33)$ & $3.107(34)$ & $3.533(39)$ & $3.056(34)$ & $3.120(34)$ & $3.472(150)$ & $*$ \\
\hline$\infty$ & 0 & $3.042(18)$ & $3.094(18)$ & $3.482(70)$ & $3.044(61)$ & $3.099(62)$ & $3.494(129)$ & $* *$ \\
\hline
\end{tabular}

TABLE II: Conventional and hybrid charmonium meson spectrum $(\mathrm{GeV})$ for the ground state from the method of Ref. 16], interpolated to the charm quark sector. The results in the continuum limit $(\beta=\infty)$ were obtained by linearly extrapolating the data to $a_{s}^{2} \rightarrow 0$. The results in the continuum limit $(*)$, with the effective masses extracted by the method of Ref. [15] are also listed. The last line $(* *)$ is the average of the results in the continuum limit from these two methods.

\begin{tabular}{|c|c|c|c|c|c|c|c|c|}
\hline$\beta$ & $a_{s}^{2}\left(f m^{2}\right)$ & $\eta_{c}$ & $J / \psi$ & $\chi_{c 1}$ & $0^{-+}$ & $1^{--}$ & $1^{++}$ & \\
\hline 2.6 & 0.0345 & $3.515(50)$ & $3.614(51)$ & $4.135(175)$ & $4.492(64)$ & $4.525(64)$ & $7.335(121)$ & \\
\hline 2.8 & 0.0236 & $3.520(60)$ & $3.625(62)$ & $4.175(183)$ & $4.379(98)$ & $4.494(77)$ & $7.333(153)$ & \\
\hline 3.0 & 0.0127 & $3.532(66)$ & $3.624(68)$ & $4.100(112)$ & $4.408(382)$ & $4.400(100)$ & $7.264(150)$ & \\
\hline$\infty$ & 0 & $3.540(102)$ & $3.633(105)$ & $4.081(205)$ & $4.335(302)$ & $4.349(148)$ & $7.237(237)$ & \\
\hline$\infty$ & 0 & $3.638(58)$ & $3.731(58)$ & $4.089(67)$ & $4.368(147)$ & $4.409(149)$ & $7.392(276)$ & $*$ \\
\hline$\infty$ & 0 & $3.589(80)$ & $3.682(81)$ & $4.085(136)$ & $4.352(225)$ & $4.379(149)$ & $7.315(257)$ & $* *$ \\
\hline
\end{tabular}

TABLE III: The same as Tab. III but for the first excited states.

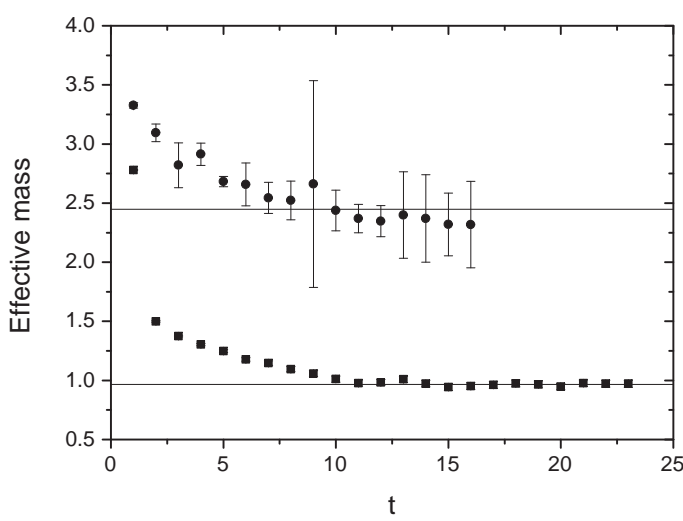

FIG. 4: Same as Fig. 3 but for the $1^{--}$hybrid meson. $a_{t} m_{1}+$ $a_{t} m_{2}$ and $a_{t} m_{1}$ are extracted respectively from the plateaux of the upper and lower curves, with $\left[t_{i}^{*}, t_{f}^{*}\right]=[6,16]$ and $\left[t_{i}, t_{f}\right]=$ $[17,23]$.

conventional charmonium with the same quantum numbers. This might mislead people into giving up further study of the non-exotic hybrids.

The last line of Tab. III shows in the continuum limit the first excited state masses of the conventional charmonium and non-exotic hybrid charmonium. The results for the conventional $0^{-+}$and $1^{--}$charmonium are in good agreement with the experimental data 3.638 and 3.686

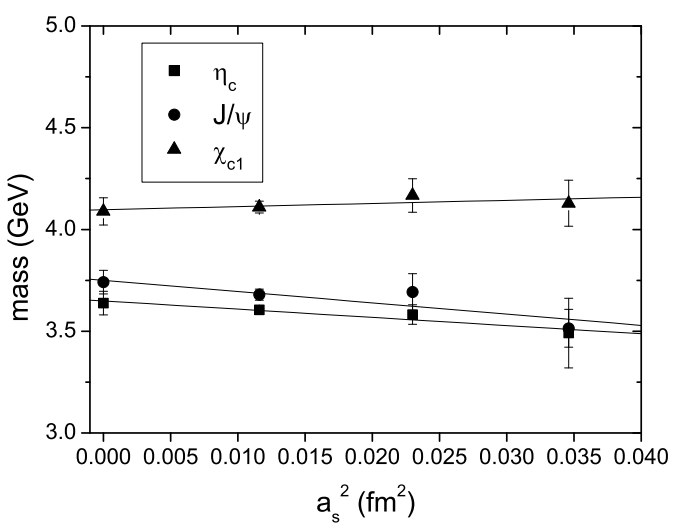

FIG. 5: Extrapolation of the excited state masses of the conventional $0^{-+}, 1^{--}$, and $1^{++}$charmonium mesons, with the effective masses extracted by the method of Ref. [15], to the continuum limit.

for $\eta_{c}(2 S)$ and $\psi(2 S)$, which supports the reliability of the methods. Although there has not been experimental input for $\chi_{c 1}\left(2^{3} P_{1}\right)$, our result is consistent with earlier lattice calculations [13, 17].

The minor differences between the data and experiments might be due to the quenched approximation used in the paper.

What new is that the first excited states of non-exotic 


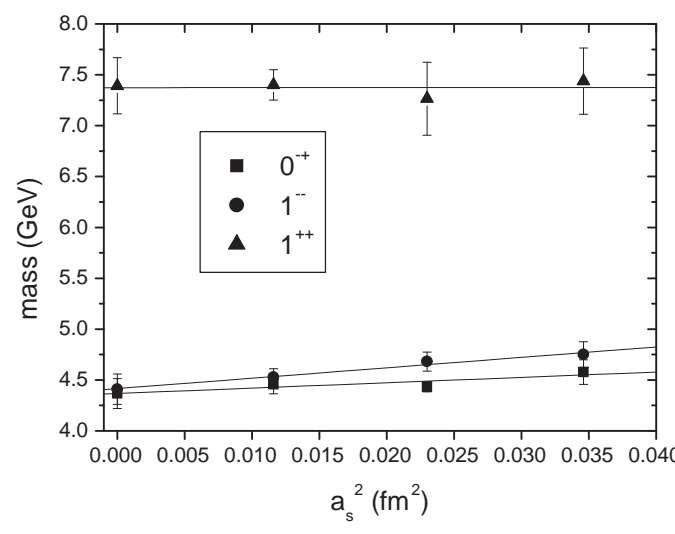

FIG. 6: The same as Fig. 5 but for the hybrid charmonium mesons.

charmonium hybrids are completely different from the conventional ones. The results in last line of Tab!II show the masses of the $0^{-+}$and $1^{--}$hybrids to be about $0.7 \mathrm{GeV}$ heavier, and the $1^{++}$about $3.2 \mathrm{GeV}$ heavier. These are very strong indications of gluonic excitations. This implies that radial excitations of the charmonium hybrids are completely different from the conventional non-hybrid ones, although their ground states overlap. This is clearly demonstrated in Figs. 16 6

This also teaches a very important lesson. One should carefully study not only the ground state, but also the excited states. Sometimes, the excited states show more fundamental properties of a hadron.

Finally, we discuss the new state $Y(4260)$, recently observed by the BaBar experiment [18] in the $J / \psi \pi^{+} \pi^{-}$ channel. It has the quantum numbers $J^{P C}=1^{--}$.

There have been several phenomenological descriptions [19, 20, 21, 22, 23, 24, 25] of this state: as tetra-quarks, a molecule of two mesons, $\psi(4 S)$, or as a hybrid meson; However, most these assumptions were not based on QCD spectrum computations.

If $Y(4260)$ is a hybrid meson, from the last line of Tab. III it could certainly not be identified as the ground state of the $1^{--}$hybrid meson. However, from our lattice QCD spectrum calculations (the last line of Tab. III), it is most probably the first excited state of the $1^{--}$hybrid charmonium. Further experimental study of the decay modes will clarify this issue.

From the last line of Tab. IIII one sees that the first excited state mass of the $0^{-+}$hybrid charmonium is about the same as that of the $1^{--}$hybrid charmonium, but much lighter than the first excited state of the $1^{++}$hybrid charmonium. It should not be very difficult to find it in future experiment.

\section{Acknowledgments}

We thank K.T. Chao, C. DeTar, E.B. Gregory, F.J. Llanes-Estrada, E. Swanson, D. Toussaint, C.Z. Yuan and S.L. Zhu for useful discussions. This work is supported by the Key Project of National Science Foundation (10235040), Project of the Chinese Academy of Sciences (KJCX2-SW-N10) and Key Project of National Ministry of Eduction (105135) and Guangdong Natural Science Foundation (05101821). We modified the MILC code [26] for simulations on the anisotropic lattice. The simulations had taken in total one year and a half on our AMD-Opteron cluster and Beijing LSSC2 XEON cluster.
[1] C. A. Meyer, AIP Conf. Proc. 698, 554 (2004).

[2] K. Peters, Int. J. Mod. Phys. A 20, 570 (2005).

[3] S. L. Olsen, J. Phys. Conf. Ser. 9, 22 (2005).

[4] D. S. Carman, arXiv:hep-ex/0511030

[5] K. Symanzik, Nucl. Phys. B 226, 187 (1983); Nucl. Phys. B 226, 205 (1983).

[6] G. Lepage and P. Mackenzie, Phys. Rev. D 48, 2250 (1993).

[7] Z. H. Mei and X. Q. Luo, Int. J. Mod. Phys. A 18, 5713 (2003).

[8] C. McNeile, Nucl. Phys. A 711 (2002) 303, and refs. theirin.

[9] C. Michael, hep-ph/0308293 and refs. theirin.

[10] Y. Liu and X. Q. Luo, arXiv:hep-lat/0511015

[11] X. Liao and T. Manke, arXiv:hep-lat/0210030

[12] C. Morningstar and M. Peardon, Phys. Rev. D 56, 4043 (1997). Phys. Rev. D 60, 034509 (1999).

[13] M. Okamoto et al. [CP-PACS Collaboration], Phys. Rev. D 65, 094508 (2002).

[14] C. Bernard et al. [MILC Collaboration], Phys. Rev. D
56, 7039 (1997).

[15] D. Guadagnoli, M. Papinutto and S. Simula, Phys. Lett. B 604, 74 (2004).

[16] C. Bernard et al., [MILC Collaboration], Phys. Rev. D 68, 074505 (2003).

[17] P. Chen, Phys. Rev. D 64, 034509 (2001).

[18] B. Aubert et al. [BABAR Collaboration], Phys. Rev. Lett. 95, 142001 (2005).

[19] S. L. Zhu, Phys. Lett. B 625, 212 (2005).

[20] F. J. Llanes-Estrada, Phys. Rev. D 72, 031503 (2005).

[21] E. Kou and O. Pene, Phys. Lett. B 631, 164 (2005).

[22] L. Maiani, V. Riquer, F. Piccinini and A. D. Polosa, Phys. Rev. D 72, 031502 (2005).

[23] X. Liu, X. Q. Zeng and X. Q. Li, Phys. Rev. D 72, 054023 (2005).

[24] F. Close and P. Page, Phys. Lett. B 628, 215 (2005).

[25] C. F. Qiao, arXiv:hep-ph/0510228

[26] http://physics.utah.edu/ detar $/ \mathrm{milc} /$ 